\title{
EFICIÊNCIA RELATIVA DE MODELOS VOLUMÉTRICOS COM E SEM A VARIÁVEL ALTURA DA ÁRVORE
}

${ }^{1}$ Júlio César Sobreira Ferreira

${ }^{2}$ José Antônio Aleixo da Silva

${ }^{3}$ Eder Pereira Miguel

${ }^{4}$ José Imaña Encinas

${ }^{5}$ José Alves Tavares

\section{RESUMO}

Foi comparada a eficiência de duas equações volumétricas, sendo uma de dupla entrada e outra baseada na medição diâmetros na primeira tora. A pesquisa foi desenvolvida em um plantio de clones de eucaliptos na Estação Experimental do Araripe, localizado no município de Araripina, no semi-árido de Pernambuco. 150 árvores foram mensuradas, sendo essas divididas em dois grupos distintos com 75 árvores para cada um. Para um grupo foram obtidos os dados de circunferência a $0,30,0,90,1,10,1,30,1,50,1,70$ e no outro grupo a $0,30,0,50,0,70$ e $1,50 \mathrm{~m}$. Em ambos os procedimentos foi cronometrado o tempo de medição. Cada árvore foi cubada, para proporcionar a obtenção do volume real através do método de Smalian. Foi feita a escolha dos dois modelos volumétricos utilizados neste estudo $\ln \mathrm{Vi}=\ln \alpha+\beta \ln \mathrm{Di}+\delta \ln \mathrm{Hi}+\xi \mathrm{i}$ (Schumacher e Hall, 1933) e $\log \mathrm{Vi}=\alpha+\beta \log \mathrm{VS} 1+\ldots+\delta$ nlogVSn $+\xi \mathrm{i}$ (Silva e Borders, 1993). Ambas as equações resultantes não diferiram estatisticamente ao nível de $1 \%$ de probabilidade do método de cubagem rigorosa de Smalian. Para estimativa de volume de árvores de eucalipto, a equação de Silva é mais aconselhável e eficiente comparada à equação de Schumacher e Hall, pois a mesma não necessita de obtenção da variável altura, diminuindo assim o tempo de obtenção de dados e os custos totais no inventário florestal.

Termos para indexação: Equações volumétricas, Eucalyptus, biometria, mensuração florestal.

${ }^{1}$ Prof. MSc. Efetivo do IFMA-Campus Codó, juliosobreira@gmail.com

2 Prof. UFRPE

${ }^{3}$ Doutorando UNB

${ }^{4}$ Prof. UNB

${ }^{5}$ Engenheiro Agrônomo IPA 


\title{
RELATIVE EFFICIENCY OF VOLUMETRIC MODELS WITH AND WITHOUT THE TREE HEIGHT VARIABLE
}

\begin{abstract}
It has been compared the efficiency of two volumetric equations, one of double entry and other based on the diameter measurement on the first log. The research was developed in a planting land of eucalyptus clones in the experimental of Araripe, located in the town of Araripina, in the semi-arid of Pernambuco. 150 trees were measured, these being divided into two different groups of 75 trees each. In one group circumference data of $0.30,0.90,1.10,1.30,1.50,1.70 \mathrm{~m}$ and in the other of $0.30,0.50,0.70$ and $1.50 \mathrm{~m}$ were obtained. In both procedures the time of measurement was recorded. Every tree was put into a cubic form, to provide the obtainment of the actual volume through the Smalian method. A choice was made of the two volumetric models used in this study $\ln$ $\mathrm{Vi}=\ln \alpha+\beta \ln \mathrm{Di}+\delta \ln \mathrm{Hi}+\xi \mathrm{i}(\mathrm{Schumacher}$ and Hall, 1933) e $\log \mathrm{Vi}=\alpha+\beta \log \mathrm{VS} 1+\ldots+$ $\delta$ nlogVSn $+\xi i$ (Silva and Borders, 1993). Both resulting equations did not statistically differ to the level of $1 \%$ of probability of Smalian's strict cubic content method. As for the estimative of the volume of eucalyptus trees, Silva's equation is more advisable and efficient compared to the equation of Schumacher and Hall, for that one does not need the obtainment of the height variable, thus reducing the time of data obtainment and the overall costs in the forest inventory.
\end{abstract}

Index terms: Volumetric equations, Eucalyptus, biometry, forest measurement. 


\section{INTRODUÇÃO}

A microrregião de Araripina, denominada de Polo Gesseiro do Araripe, localizada no semi-árido de Pernambuco, tem como sua principal fonte econômica a exploração de gipsita, com produção anual de aproximadamente 2,6 milhões de ton/ano de gesso, correspondendo a $94 \%$ de todo o gesso consumido no Brasil (SALDANHA et al., 2007).

Para a produção de gesso na região, as indústrias necessitam cada vez mais de energia para suprir sua demanda nos fornos de calcinação da gipsita, matéria-prima usada na obtenção do gesso. Em função da inviabilidade econômica de outras fontes energéticas como coque, óleo BPF e energia elétrica, as reservas florestais da região estão sendo destruídas pelo crescente consumo de lenha.

A transformação da vegetação nativa em lenha para as indústrias do gesso foi e continua sendo a principal causa da devastação florestal na área do Araripe. Segundo Sá et al. (2007), para se produzir uma tonelada de gesso se consome, aproximadamente, 0,7 st de madeira nativa. Isso implica em uma demanda de madeira de $1.900 .000 \mathrm{st} / \mathrm{ano}$.

A área do semi-árido inserida no polígono das secas ainda não dispõe de informações básicas e indispensáveis sobre o comportamento das espécies florestais de rápido crescimento que podem se adaptar à região sem causar alterações nas condições ambientais. Tais florestas poderiam minimizar os impactos ambientais de caráter negativos, provocados, principalmente, pela ação antrópica na busca de material combustível para atender a demanda de energia.

Uma alternativa de conter a devastação do estoque lenhoso da região seria a utilização de madeira de florestas de rápido crescimento, porém, faz-se necessário o conhecimento da produtividade dessas florestas. Dentre as espécies de rápido crescimento, o eucalipto se destaca por sua alta produtividade e múltipla versatilidade na utilização da madeira produzida (SIMÕES, 1970; GONÇALEZ et al., 2006).

Efetuando projeções a partir dos dados da Sociedade Brasileira de Silvicultura (2005), estima-se que atualmente existam cerca de quatro milhões de florestas plantadas com eucaliptos no Brasil. Por ser uma espécie que se adaptou bem às diferentes regiões do Brasil, atualmente, o país é visto como o maior produtor de Eucalyptus do mundo (SARTORETTO et al., 2008).

O conhecimento e estudo sobre volume de madeira constituem hoje em dia um dos itens mais importantes quando se trata de assuntos relacionados à utilidade da madeira em vários setores industriais, como também é de grande utilidade nos trabalhos de inventário. Machado et al. (2005) afirma que o volume facilita o conhecimento do 
potencial florestal em uma região, proporcionando assim uma ferramenta poderosa na avaliação do conteúdo lenhoso dos povoamentos florestais.

De acordo com Oliveira et al. (2005) o uso de equações volumétricas em povoamentos florestais proporciona rapidez, precisão e simplicidade, mostrando que esse tipo de método pode ser tão preciso quanto o inventário florestal convencional. Segundo Couto e Bastos (1987) o uso de equações volumétricas apresenta a vantagem de calcular o volume sólido, árvore a árvore, através de modelos matemáticos, especialmente testados para apresentar os menores erros possíveis, sem a necessidade do abate da árvore.

Dentre as equações volumétricas existentes na literatura florestal, as regionais, que se baseiam em medir a altura, o diâmetro e a altura do peito (DAP), são as mais usuais, no entanto utilizam estimativas de alturas de árvores que, dependendo da habilidade do operador, pode se constituir em uma considerável fonte de erro.

Dos vários tipos de modelos volumétricos, o de Schumacher e Hall tem se destacado na estimativa do volume de árvores e de povoamentos florestais (SCOLFORO et al., 2004; SANTANA et al., 2005; THOMAS et al., 2006). O modelo considera como variáveis independentes o DAP e a altura da árvore $(\mathrm{H})$.

Silva et al. (1992) e Silva e Borders (1993) afirmam que modelos volumétricos que dispensam a medição da variável altura das árvores para estimativa de volume, podem obter volumes em várias secções da primeira tora da árvore. Esses modelos se baseiam em medições de volumes parciais na primeira tora que vai de 0,30 a $1,70 \mathrm{~m}$.

Uma maneira eficiente de comparar diferentes procedimentos de obtenção de modelos volumétricos é o teste da eficiência relativa (ER\%). De acordo com Silva et al. (1997) o teste além de considerar medidas de variação presentes nas equações, também leva em conta o tempo de operação, ou seja, busca encontrar a equação ideal que vise obter maior precisão com baixos custos.

Baseando nesses argumentos, o objetivo deste trabalho foi comparar a eficiência de duas equações volumétricas, sendo uma de dupla entrada (Schumacher e Hall) e outra baseada em volumes na primeira tora (Silva) quando comparadas com o volume real. Os tempos de mensuração dos dois métodos, bem como o da cubagem rigorosa pelo método de Smalian foram cronometrados, a fim de calcular a eficiência relativa em percentagem. 


\section{MATERIAL E MÉTODOS}

\section{Localização}

A pesquisa foi desenvolvida na Estação Experimental do Araripe, pertencente ao Instituto Agronômico de Pernambuco (IPA), localizada no município de Araripina, nas coordenadas geográficas $07^{\circ} 29^{\prime} 00^{\prime} \mathrm{S}$ e $40^{\circ} 36^{\prime} 00^{\prime} \mathrm{W}$ e altitude de 816 metros.

O clima regional predominante na região é do tipo BShw` pela classificação de Köppen, quente e seco com chuvas de verão, apresentando precipitação de $763 \mathrm{~mm}$ anuais. A temperatura é de 22,4o C, com máxima de 30,3o C e mínima de 20,6o C (ARAÚJO, 2004). O solo é do tipo latossolo vermelho-amarelo

\section{Seleção das árvores a serem cubadas}

As árvores selecionadas fazem parte de um experimento composto por 15 clones de eucaliptos, plantados no espaçamento $2 \times 3 \mathrm{~m}$.

Cada parcela media 14 × 21 metros, ocupando área de 294 m2, com 49 árvores por parcelas. Eliminando a bordadura, a parcela de amostragem teve 25 árvores (Figura $1)$.

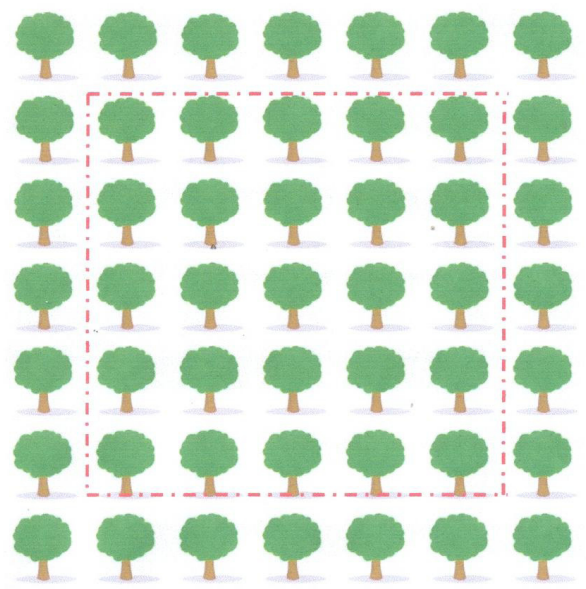

$14 \mathrm{x} 21 \mathrm{~m}$

Figura 1. Dimensões da parcela e localização das árvores medidas

Foram selecionadas e cubadas 150 árvores. Sendo essas divididas em dois grupos distintos, A e B, com 75 árvores para cada grupo, utilizando-se do processo de amostragem inteiramente aleatório.

Cada árvore foi derrubada e medida em diferentes alturas, suas circunferências 
e seu comprimento total para proporcionar assim a obtenção do volume real através do método de Smalian, a partir da expressão matemática:

$$
\begin{aligned}
& \mathbf{V}_{\mathbf{s}}=\frac{A_{1}+A_{2}}{2} \cdot L \\
& \mathbf{V}_{\mathbf{t}}=\sum_{i=1}^{n} V_{s}
\end{aligned}
$$

Em que: $\mathrm{V}_{\mathrm{t}}=$ Volume total em $\mathrm{m}^{3}$;

$\mathrm{V}_{\mathrm{s}}=$ Volume da secção ;

$\mathrm{A}=$ Área da secção

Os modelos utilizados nesta pesquisa foram:

$$
\begin{array}{ll}
\ln \mathrm{Vi}=\ln \alpha+\beta \ln \mathrm{D}_{\mathrm{i}}+\delta \ln \mathrm{H}_{\mathrm{i}}+\xi^{\mathrm{i}} & \text { (Schumacher e Hall, 1933 } \\
\log \mathrm{Vi}=\pi+\beta \log \mathrm{S}_{1 \mathrm{i}}+\ldots+\delta_{\mathrm{n}} \log \mathrm{VS}_{\mathrm{ni}}+\xi_{\mathrm{i}} & \text { (Silva e Borders, 1993) }
\end{array}
$$

Em que:

$\mathrm{V}_{\mathrm{i}}=$ Volume da árvore $\mathrm{i}$;

$\mathrm{D}_{\mathrm{i}}=$ diâmetro à altura do peito, $(\mathrm{DAP})$ da árvore $\mathrm{i}$;

$\mathrm{H}=$ Altura total da árvore $\mathrm{i}$;

$\beta, \alpha, \delta=$ Coeficientes dos modelos;

$\mathrm{VS}=$ volume da secção na primeira tora com comprimento de $1,70 \mathrm{~m}$; $\xi=$ Erro aleatório.

O procedimento Stepwise (DRAPER e SMITH, 1981) foi aplicado no modelo linear Silva e Borders, que utiliza mais de uma variável independente. Sendo consideradas as 28 variações de tamanhos de seções medidas no intervalo de 0,30 m a 1,70 m (Tabela 1). No teste de seleção das variáveis independentes, usou-se o nível de probabilidade de $\mathrm{F}$ inferior a $1 \%$. 
Tabela 1. Vinte e oito combinações de secções da primeira tora, utilizadas na avaliação dos volumes de árvores de Eucalyptus, em inventário realizado no Módulo de Experimentação Florestal para a Região do Araripe, em Araripina-PE.

\begin{tabular}{cccccc}
\hline Secção & $\begin{array}{c}\text { Intervalo do } \\
\text { tronco }(\mathrm{m})\end{array}$ & $\begin{array}{c}\text { Comprimento } \\
(\mathrm{m})\end{array}$ & Secção & $\begin{array}{c}\text { Intervalo do } \\
\text { tronco }(\mathrm{m})\end{array}$ & $\begin{array}{c}\text { Comprimento } \\
(\mathrm{m})\end{array}$ \\
\hline 1 & 0,30 a 0,50 & 0,2 & 15 & 0,70 a 1,10 & 0,4 \\
2 & 0,30 a 0,70 & 0,4 & 16 & 0,70 a 1,30 & 0,6 \\
3 & 0,30 a 0,90 & 0,6 & 17 & 0,70 a 1,50 & 0,8 \\
4 & 0,30 a 1,10 & 0,8 & 18 & 0,70 a 1,70 & 1,0 \\
5 & 0,30 a 1,30 & 1,0 & 19 & 0,90 a 1,10 & 0,2 \\
6 & 0,30 a 1,50 & 1,2 & 20 & 0,90 a 1,30 & 0,4 \\
7 & 0,30 a 1,70 & 1,4 & 21 & 0,90 a 1,50 & 0,6 \\
8 & 0,50 a 0,70 & 0,2 & 22 & 0,90 a 1,70 & 0,8 \\
9 & 0,50 a 0,90 & 0,4 & 23 & 1,10 a 1,30 & 0,2 \\
10 & 0,50 a 1,10 & 0,6 & 24 & 1,10 a 1,50 & 0,4 \\
11 & 0,50 a 1,30 & 0,8 & 25 & 1,10 a 1,70 & 0,6 \\
12 & 0,50 a 1,50 & 1,0 & 26 & 1,30 a 1,50 & 0,2 \\
13 & 0,50 a 1,70 & 1,2 & 27 & 1,30 a 1,70 & 0,4 \\
14 & 0,70 a 0,90 & 0,2 & 28 & 1,50 a 1,70 & 0,2 \\
\hline
\end{tabular}

Obtendo-se os volumes reais a partir da fórmula de Smalian. Os modelos foram ajustados pela análise de regressão utilizando o programa Systat. Utilizou-se para comparação o coeficiente de determinação ajustado (R2aj) expresso por:

$$
\mathrm{R}^{2}{ }_{\mathrm{aj}}=\mathrm{R}^{2}-\left(1-\mathrm{R}^{2}\right)\left(\frac{n-1}{n-p}\right)
$$

Em que:

$\mathrm{p}=$ Número de parâmetros no modelo;

$\mathrm{n}=$ Número de observações;

$\mathrm{R}_{\text {aj }}^{2}=$ Coeficiente de determinação ajustado.

Também se considerou a distribuição percentual dos resíduos, expresso por:

$$
R_{v}(\%)=\frac{V_{\text {real }}-V_{\text {est }}}{V_{\text {real }}} \times 100
$$

Em que:

Vreal $=$ Volume real das árvores cubadas rigorosamente;

Vest $=$ Volume estimado das árvores através das equações volumétricas.

Os volumes estimados a partir das equações ajustadas foram obtidos para cada 
uma das árvores.

Realizaram-se análises da variância (ANOVA) para cada grupo, para verificar a existência ou não de diferença significativa entre as equações volumétricas testadas.

Foi utilizado o delineamento inteiramente aleatório, com três tratamentos para volume (Volume real cubado por Smalian, volume estimado pela equação de Silva e volume estimado pela equação de Schumacher e Hall). Todos os procedimentos foram cronometrados.

Para o cálculo da eficiência relativa (ER\%) das equações volumétricas, aplicouse a expressão (SILVA et al., 1997):

$$
\mathrm{ER} \%=\frac{\bar{T}_{i} \times\left(\mathrm{CV}_{\mathrm{i}}\right) \times\left|\mathrm{R}_{\mathrm{vi}} \%\right|}{\overline{T_{j}} \times\left(\mathrm{CV}_{\mathrm{j}}\right) \times\left|\mathrm{R}_{\mathrm{vj}} \%\right|} \times 100
$$

Em que:

$\mathrm{ER} \%=$ Eficiência relativa;

$\bar{T}_{i}=$ Tempo médio de medição para a equação de Schumacher e Hall;

$\overline{T_{j}}=$ Tempo médio de medição para equação de Silva;

$\mathrm{CVi}=$ Coeficiente de variação da equação de Schumacher e Hall;

$\mathrm{CVj}=$ Coeficiente de variação da equação de Silva;

Rvi\% = Resíduo em percentagem da equação de Schumacher e Hall;

$\mathrm{Rvj} \%=$ Resíduo em percentagem da equação de Silva.

Segundo Silva et al., (1997) quando o valor de ER\% for menor que 100, a equação localizada no numerador é mais eficiente do que a equação localizada no denominador, e vice-versa. Porém, se o ER\% for igual ou próximo de 100, assume-se que as equações não se diferem uma da outra, sendo assim semelhantes.

\section{RESULTADOS E DISCUSSÃO}

O procedimento Stepwise aplicado no modelo linear de Silva e Borders que possuem mais de uma variável independente nos dois grupos A e B, apresentou o seguinte resultado: Para o grupo A o modelo de Silva e Borders que apresentava 28 combinações (secções) passou a ter apenas três $(6,19,26)$; o grupo B também obteve três combinações $(1,6,8)$, no qual cada combinação possuía duas medidas (Tabela 2). 
Tabela 2. Resultado do procedimento Stepwise, para estimativa do volume de clones de Eucalyptus para o modelo de Silva - Borders nos grupos A e B.

\begin{tabular}{|c|c|c|}
\hline Grupos & Silva - Borders antes & Silva - Borders depois \\
\hline A & $\begin{array}{l}\log \mathrm{V}_{\mathrm{i}}=\alpha+\beta \log \mathrm{VS}_{1 \mathrm{i}}+\ldots+ \\
\delta_{\mathrm{n}} \log \mathrm{VS}_{\mathrm{ni}}+\xi_{\mathrm{i}}\end{array}$ & $\begin{array}{l}\ln V_{i}=\alpha+\beta \ln \left(0,4712\left(D^{2}{ }_{0,30}+D^{2}{ }_{1,50}\right)\right)+\delta \ln \left(0,0785\left(D^{2}{ }_{0,90}+\right.\right. \\
\left.\left.D^{2}{ }_{1,10}\right)\right)+\rho \ln \left(0,1571\left(D_{1,30}^{2}+D_{1,50}^{2}\right)\right.\end{array}$ \\
\hline B & $\begin{array}{l}\log \mathrm{V}_{\mathrm{i}}=\alpha+\beta \log \mathrm{VS}_{1 \mathrm{i}}+\ldots+ \\
\delta_{\mathrm{n}} \log \mathrm{VS}_{\mathrm{ni}}+\xi_{\mathrm{i}}\end{array}$ & $\begin{array}{l}\ln V_{i}=\alpha+\beta \ln \left(0,0785\left(D_{0,30}^{2}+D_{0,50}^{2}\right)\right)+\delta \ln \left(0,4712\left(D_{0,30}^{2}+\right.\right. \\
\left.\left.D^{2}{ }_{1,50}\right)\right)+\rho \ln \left(0,0785\left(D_{0,50}^{2}+D_{0,70}^{2}\right)\right.\end{array}$ \\
\hline
\end{tabular}

Após ajuste dos modelos, obtiveram-se os seguintes coeficientes para as equações de Schumacher e Hall e Silva e Borders (Tabelas 3 e 4).

Tabela 3. Coeficientes das equações no grupo A.

\begin{tabular}{lllllll}
\hline Equação & \multicolumn{3}{c}{ Coeficientes } & $\mathrm{R}_{\mathrm{a}}{ }^{2} \%$ & $\mathrm{R}_{\mathrm{v}} \%$ \\
\hline & $\mathrm{b}_{0}$ & $\mathrm{~b}_{1}$ & $\mathrm{~b}_{2}$ & $\mathrm{~b}_{3}$ & & \\
\cline { 2 - 7 } 1 & $-0,0975$ & 1,8938 & 0,5880 & & 96,8 & $-0,29$ \\
2 & 3,0347 & 0,1993 & $-0,2382$ & 1,1394 & 96,6 & $-0,26$ \\
\hline
\end{tabular}

Tabela 4. Coeficientes das equações no grupo B.

\begin{tabular}{|c|c|c|c|c|c|c|}
\hline Equação & & Coe & entes & & $\mathrm{R}_{\mathrm{a}}{ }^{2} \%$ & $\mathrm{R}_{\mathrm{v}} \%$ \\
\hline & $\mathrm{b}_{0}$ & $\mathrm{~b}_{1}$ & $\mathrm{~b}_{2}$ & $b_{3}$ & & \\
\hline 1 & 0,7306 & 2,1034 & 0,4463 & & 96,5 & 0,43 \\
\hline 2 & 2,6891 & $-0,0696$ & 0,8495 & 0,3640 & 93,1 & $-0,41$ \\
\hline
\end{tabular}

foram:

Com base nos coeficientes obtidos para cada grupo, as equações resultantes Para o grupo A:

1- $\operatorname{Ln} \mathrm{V}_{\mathrm{i}}=-0,0975+1,8938 \times \ln (\mathrm{DAP})+0,588 \times \ln (\mathrm{H})($ Schumacher e Hall)

2- $\operatorname{Ln} \mathrm{V}_{\mathrm{i}}=3,0347+0,1993 \times \ln \left(0,4712 \times\left(\mathrm{D}_{0,30}^{2}+\mathrm{D}_{1,50}^{2}\right)\right)-0,2382 \times \ln (0,0785$ $\left.\mathrm{x}\left(\mathrm{D}_{0,90}^{2}+\mathrm{D}_{1,10}^{2}\right)\right)+1,1394 \mathrm{x} \ln \left(0,1571 \times\left(\mathrm{D}_{1,30}^{2}+\mathrm{D}_{1,50}^{2}\right)\right)($ Silva e Borders

Para o grupo B:

1- $\operatorname{Ln} \mathrm{V}_{\mathrm{i}}=0,7306+2,1034 \mathrm{x} \ln (\mathrm{DAP})+0,4463 \times \ln (\mathrm{H})($ Schumacher e Hall)

2- $\operatorname{Ln} \mathrm{V}_{\mathrm{i}}=2,6891-0,0696 \times \ln \left(0,0785 \times\left(\mathrm{D}_{0,30}^{2}+\mathrm{D}_{0,50}^{2}\right)\right)+0,8495 \times \ln (0,4712$ $\left.\mathrm{x}\left(\mathrm{D}_{0,30}^{2}+\mathrm{D}_{1,50}^{2}\right)\right)+0,3640 \mathrm{x} \ln \left(0,0785 \mathrm{x}\left(\mathrm{D}_{0,50}^{2}+\mathrm{D}_{0,70}^{2}\right)\right)$ (Silva e Borders) 
Verificou-se que foram obtidas estimativas precisas nas equações, devido tanto aos altos valores dos coeficientes de determinação ajustados quanto aos baixos valores do resíduo.

Após a realização das análises de variâncias, tanto para o grupo A, como para o grupo B, pode-se verificar que não houve diferença significativa ao nível de $1 \%$ de probabilidade para as equações testadas (Tabelas 5 e 6 ).

Tabela 5. Análise da variância dos volumes para o grupo A.

\begin{tabular}{lcccc}
\hline \multicolumn{1}{c}{ FV } & GL & SQ & QM & F \\
\hline Tratamentos & 2 & 0,00000435 & 0,00000218 & $0,0015^{\text {ns }}$ \\
Resíduo & 222 & 0,33256 & 0,00150 & \\
\hline Total & 224 & 0,33256 & & \\
\hline
\end{tabular}

Tabela 6. Análise da variância dos volumes para o grupo B.

\begin{tabular}{lcccc}
\hline \multicolumn{1}{c}{ FV } & GL & SQ & QM & F \\
\hline Tratamentos & 2 & 0,00013 & 0,00007 & $0,0504^{\text {ns }}$ \\
Resíduo & 222 & 0,28793 & 0,00130 & \\
\hline Total & 224 & 0,28806 & & \\
\hline
\end{tabular}

Ns $=$ não significativo $(\mathrm{p}>=0.01)$

Em que:

$\mathrm{FV}=$ Fonte de variação;

$\mathrm{GL}=$ Grau de liberdade;

$\mathrm{SQ}=$ Soma de quadrados;

$\mathrm{QM}=$ Quadrado médio;

$\mathrm{F}=$ Estatística do teste $\mathrm{F}$.

Os volumes reais calculados pelo método de Smalian e os volumes estimados pelas equações Silva e Borders e Schumacher e Hall não diferiram entre si.

Com esses resultados, as duas equações podem ser usadas no campo para a estimativa de volume, porém a equação de Schumacher e Hall, que utiliza como variável independente a altura que é medida através de instrumentos óticos, torna o trabalho de campo mais demorado e cansativo em termo de operacionalidade, principalmente se as copas das árvores se tocarem, o que também pode resultar em erros.

Os tempos médios para obtenção dos dados no campo pelo método de Silva e Borders e Schumacher e Hall estão nas (Tabelas 7 e 8). 
Tabela 7. Tempo médio gasto para obtenção dos dados do grupo A para as equações Silva e Borders e Schumacher e Hall com seus coeficientes de variações

\begin{tabular}{ccc}
\hline $\begin{array}{c}\text { Tempo médio de medição para o } \\
\text { grupo A }\end{array}$ & Segundos & $\begin{array}{c}\text { Coeficiente de Variação } \\
(\mathrm{CV}) \%\end{array}$ \\
\hline Silva & 51 & 27,81 \\
\hline Schumacher e Hall & 107 & 42,65 \\
\hline Diferença média por árvore & 56 & \\
\hline & & \\
\hline
\end{tabular}

Tabela 8. Tempo médio gasto para obtenção dos dados do grupo B para as equações Silva e Borders e Schumacher e Hall com seus coeficientes de variações

\begin{tabular}{ccc}
\hline $\begin{array}{c}\text { Tempo médio de medição para o } \\
\text { grupo B }\end{array}$ & Segundos & $\begin{array}{c}\text { Coeficiente de Variação } \\
(\mathrm{CV}) \%\end{array}$ \\
\hline Silva & 28 & 31,05 \\
\hline Schumacher e Hall & 72 & 38,54 \\
\hline Diferença média por árvore & 44 & \\
\hline
\end{tabular}

A diferença média do tempo para a obtenção dos dados das equações Silva e Borders e Schumacher e Hall foi de 56 segundo para o grupo A e 44 segundos para o grupo B, ou seja, esse é o tempo médio que se gasta a mais coletando dados de uma só árvore, para a equação de Schumacher e Hall em comparação com a equação de Silva e Borders.

Esses resultados evidenciam que pela equação de Silva e Borders, o trabalho de coleta de dados se torna mais rápido, devido a mesma não necessitar do deslocamento do operador da árvore, já que as medidas dos dados necessários do modelo proposto é feita diretamente na árvore. Já para a equação de Schumacher e Hall o tempo foi maior devido ao deslocamento do operador da árvore para obtenção da altura, que em alguns casos, perde-se um maior tempo procurando uma melhor visualização da copa da árvore para utilização correta do hipsômetro.

Esses resultados corroboram com os obtidos na pesquisa desenvolvida por Silva(1992) e por Silva e Borders(1993).

Os coeficientes de variação se mostraram elevados para as duas equações, isso ficou evidenciado devido à ocorrência de pequenas oscilações de tempo na obtenção dos dados em algumas árvores, por causa das dificuldades do local onde a árvore se encontrava no campo. 
Os resultados do cálculo da eficiência relativa nas equações, para poder provar e comprovar qual melhor equação a ser utilizada em campo, estão evidenciados nas tabelas 9 e 10 .

Tabela 9. Resultados das eficiências relativas (\%) da equação de Silva e Borders comparada a equação de Schumacher e Hall nos grupos A e B.

\begin{tabular}{cc}
\hline Eficiência relativa (\%) & Silva \\
\hline Schumacher e Hall A & 238,93 \\
\hline Schumacher e Hall B & 288,76 \\
\hline
\end{tabular}

Tabela 10. Resultados das eficiências relativas (\%) da equação de Schumacher e Hall comparada a equação de Silva e Borders nos grupos A e B.

\begin{tabular}{cc}
\hline Eficiência relativa (\%) & Schumacher e Hall \\
\hline Silva A & 41,85 \\
\hline Silva B & 34,63 \\
\hline
\end{tabular}

Conforme os resultados apresentados da eficiência relativa, pode-se observar que a equação de Silva e Borders, no grupo A é 138,93\% mais eficiente do que a equação de Schumacher e Hall, que se mostrou somente $41,85 \%$ de eficiência quando comparada à equação de Silva e Borders no mesmo grupo.

Já para o grupo B, Silva e Borders se mostra 188,76\% mais eficiente que a equação de Schumacher e Hall, que também demonstrou-se com baixa eficiência de $34,63 \%$, quando comparada à equação de Silva e Borders.

Assim, por suas estimativas não diferirem do método de cubagem rigorosa e por apresentarem uma maior eficiência relativa que o método de Schumacher e Hall, a equação de Silva e Borders pode ser usada com boa precisão na estimativa volumétrica de clones de eucaliptos na chapada do Araripe.

\section{CONCLUSÃO}

Para estimativa de volume de árvores de eucalipto, a equação de Silva e Borders é mais aconselhável e eficiente, quando comparada à equação de Schumacher e Hall, pois a mesma não necessita de obtenção da variável altura, diminuindo assim o tempo de obtenção de dados, ocasionando a redução de custos totais no trabalho. 


\section{REFERÊNCIAS}

ARAÚJO, S.M.S. O Pólo Gesseiro do Araripe: Unidades geo-ambientais e impactos da mineração. Tese (Doutorado em Ciências, Área de Administração e Política de Recursos Minerais). Campinas. UNICAMP,2004. 276p.

COUTO, H.T.Z.; BASTOS, N.L.M. Modelos de equações de volume e relações hipsométricas para plantações de Eucalyptus no Estado de São Paulo. IPEF, n.37, p.3334, 1987.

GONÇALEZ, J.C.; et al. Características tecnológicas das madeiras de Eucalyptus grandis W. Hill ex Maiden e Eucalyptus cloeziana F. Muell visando ao seu aproveitamento na indústria moveleira. Ciência Florestal, vol.16, n.3, p. 329-341, 2006.

MACHADO, S.A.; URBANO, E.; CONCEIÇÃO, M.B. Comparação de métodos de estimativa de volume para Pinnus oocarpa em diferentes idades e diferentes regimes de desbastes. Bol. Pesq. Fl. Colombo, n.50, p. 81-98 Jan/Jun. 2005.

OLIVEIRA, M.L.R.; et al. Equações de volume de povoamento para fragmentos florestais naturais do município de Viçosa, Minas Gerais. Viçosa-MG, Revista árvore, v.29, n.2, p.213-225, 2005.

SÁ, I.B; SÁ, I.I.S; SILVA, D.F. Geotecnologias conciliando preservação ambiental e fortalecimento das atividades produtivas na região do Araripe-PE. In: Anais XIII Simpósio Brasileiro de Sensoriamento Remoto, Florianópolis, Brasil, INPE p. 1875-1882, 2007.

SALDANHA, E.C.M.; et al Uso do gesso mineral em latossolo cultivado com cana de açúcar. (Mossoró-Brasil), Caatinga v.20, n.1, p.36-42, jan-mar, 2007.

SANTANA, C; et al. Sistema de equações para simulação do crescimento e da produção em povoamentos de Eucalyptus grandis Hill ES. Sem desbaste baseado no modelo de Clutter. Guarapuava, PR, Ambiência, v.1 n.2, p. 239-256, 2005.

SARTORETTO, L.M.; SALDANHA, C.W.; CORDER, M.P.M. Transformação genética: estratégias e aplicações para o melhoramento genético de espécies florestais. Santa Maria, Ciência Rural, v.38, n.3, p.861-871, mai-jun, 2008.

SBS, 2005. Disponível em: (http://www.sbs.com.br) > Acesso em: 22 de abril de 2008.

SCOLFORO,J.R.S.; et al. Estimativa de volume, peso seco, peso de óleo e quantidade de moirões para a candeia (Eremanthus erythropappus (DC). Macleish). Lavras, Cerne, V.10 n.1, p.87-102, 2004. 
SILVA, J.A.A. et al. A tree volume equation base on two lower stem diameters for Caribbean Pine in Sri Lanka. Oxford, Commonwealth Forestry Review, v.71, n.225,p. 114-116, June, 1992.

SILVA, J.A.A.; BORDERS, B.E. A tree volume equation based on two lower, stem diameters for Loblolly Pine in the Southeastern United States. Washington, US, Southern Journal of Applied Forestry, v.17, n.4, p. 160-162, 1993.

SILVA, J.A.A.; et al. Estimativa da suficiência amostral para avaliar intensidade de infestação da Diatraea spp.em cana-de-açúcar. Pesq.Agropec.Bras., Brasília, v.32, n.10, p.1003-1007, out. 1997.

SILVA, S.M.F.S. Comparação entre equações volumétricas regionais e equações baseadas em volume da primeira tora em clones de Eucalyptus na Chapada do Araripe-PE. Dissertação (Mestrado em Ciências Florestais) Universidade Federal Rural de Pernambuco. Departamento de Ciência Florestal. f. 58. 2008.

SIMÕES, J.W. Método de produção de mudas de eucalipto. IPEF, n.1, p.101-116, 1970.

SCHUMACHER, F; HALL, F. Logarithmic expression of timber-tree volume. Journal of Agricultural Research, v.47, p. 719-734, 1933.

THOMAS, C.; et al. Comparação de equações volumétricas ajustadas com dados de cubagem e análise de tronco. Santa Maria, Ciência Florestal, v.16, n.3, p.319-327, 2006.

TROVÃO, D.M.B.M.; et al. Avaliação do potencial hídrico de espécies da Caatinga sob diferentes níveis de unidade no solo. Revista de Biologia e Ciência da Terra, v. 4, n.2, 2004. 